\title{
A DECOMPOSITION OF STRONG GROUND MOTION
}

\author{
W. R. Stephenson'
}

\begin{abstract}
The Cedar Hills Nursery strong motion record of the 1987 October 1st Whittier Narrows earthquake can be decomposed into five physically distinct components. These are the usual radial, circumferential and vertical components, plus a $2.84 \mathrm{~Hz}$ resonance along $317^{\circ} \mathrm{N}$ and a 3.27 $\mathrm{Hz}$ resonance along $317^{\circ} \mathrm{N}$. The significance and possible cause of the resonant parts are discussed.
\end{abstract}

\section{INTRODUCTION}

Among the strong motion records generated by the 1987 October 1st Whittier Narrows (California) earthquake, the Cedar Hills Nursery, Tarzana, record stands out as being resonant and having much higher ground accelerations than any other record in the vicinity. However, smaller events recorded at the same site (aftershock, October 4, 1987; Pasadena, December 3, 1988; Upland, February 28, 1990; Sierra Madre, June 28,1991 ) have not been described as showing this abnormal behaviour. Resonance occurs when a mechanical system is subjected to an oscillating force at a frequency close to its own natural frequency, and it results in abnormally large, nearly sinusoidal responses. A possible reason for the lack of resonant response during the smaller events is that they may not have supplied energy at the resonant frequencies, but this cannot be checked because no nearby measurements of rock motion were made.

When the true three-dimensional nature of site response is considered, another reason becomes possible for the excitation of resonances in some events but not in others. The strong ground motion generated by an earthquake is a superposition of various seismic phases, each with its own wavefronts and particle motions, with each phase exciting ground resonances by a particular mechanism. Thus each earthquake will excite resonances according to the phases arriving at a site. By way of illustration, a vertically propagating plane wave will easily be able to excite a classic infinite horizontal layer resonance, but less able to excite resonances which involve torsion about a vertical axis.

In structural engineers' words, each resonance has a mode shape and a participation factor. These have equivalents in resonant site-response studies, where each vibrational mode shape can be excited differently by various motions even if those motions have the same spectra.

1 Institute of Geological and Nuclear Sciences, Wellington (Member)
The possibility that different phases of ground motion vary in their ability to excite ground resonances is not one to be dismissed lightly, for it challenges the idea that small earthquakes or microtremors may be used to characterise the resonant character of sites. Any systematic change with earthquake magnitude, of the energy carried in different phases, would devalue such an approach.

\section{DECOMPOSITION}

The Cedar Hills Nursery accelerogram shown in Figure 1 was recorded at a distance of $43 \mathrm{~km}$ from the epicentre of the Whittier Narrows earthquake, which was at an azimuth of $105^{\circ}$ N.

Auto and cross power spectra of the horizontal motions of Figure 1 show that there are sharp resonances at $2.84 \mathrm{~Hz}$ and $3.27 \mathrm{~Hz}$ and that these resonances act along the direction $317^{\circ}$ $\mathrm{N}$ It is therefore reasonable that these two directed resonances should be considered separately, and that the ground motion should be decomposed into the five components shown in Figure 2. This decomposition was achieved by a process of record rotation, spectral analysis, and fitting of windowed sinusoids, and will be described elsewhere. It suffices for the purposes of this note to confirm that the vector sum of the components (a) to (e) gives the original record, and that the components (d) and (e) each have a simple resonant nature and act along a single direction $\left(317^{\circ} \mathrm{N}\right)$

Concentrating now on the components (d) and (e), it can be seen that their histories are very different, even though their frequencies differ by only $15 \%$. However if both responses were excited by the same wave fronts and particle orbits they would be expected to have much the same response histories because the input spectra would be expected to vary similarly with time. No nearby rock records are available for this earthquake so the variation of input spectrum with time cannot be checked. 


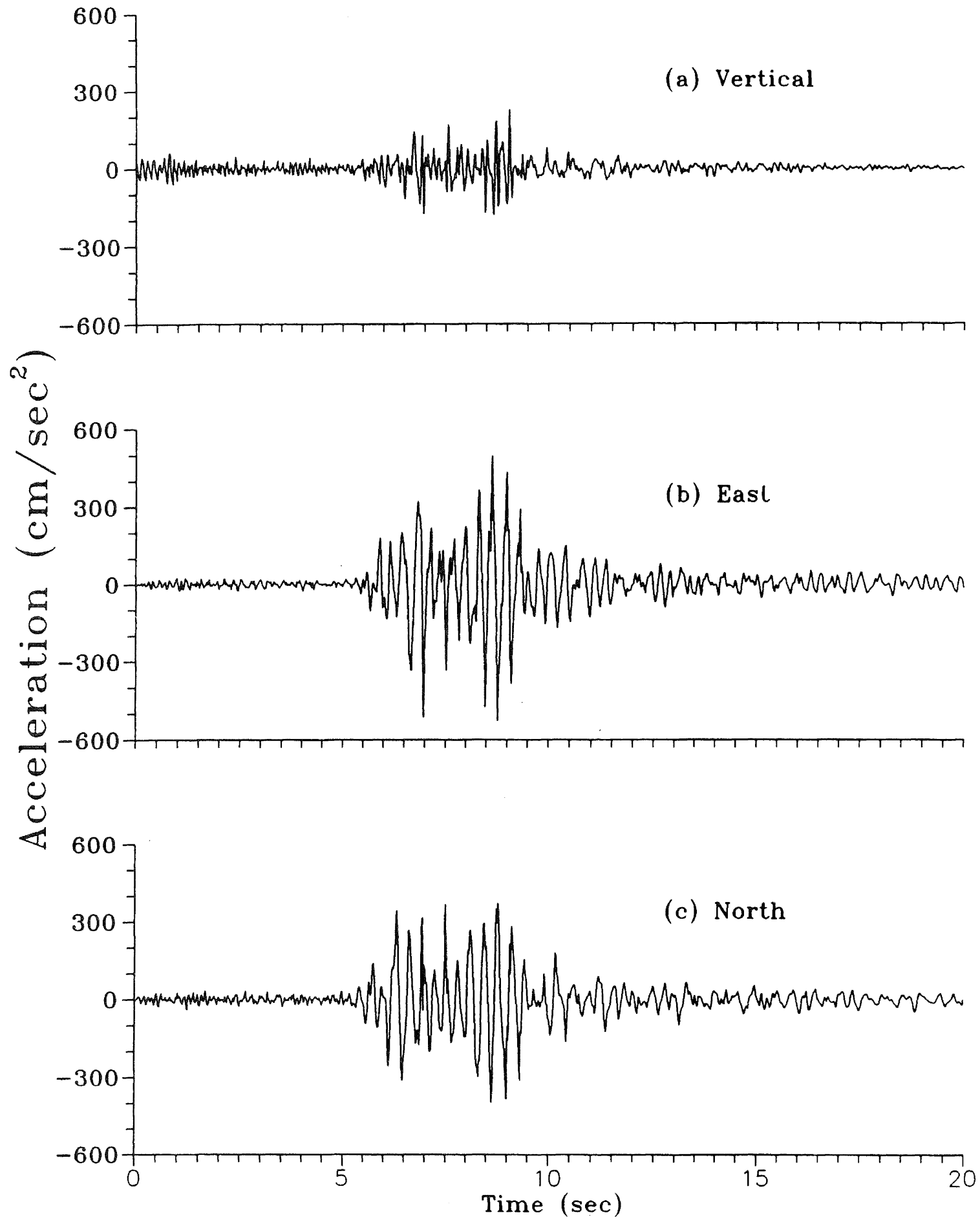

Figure 1 The original accelerogram recorded during the 1987 October 1 Whittier Narrows earthquake at the Cedar Hills Nursery, Tarzana, site. 

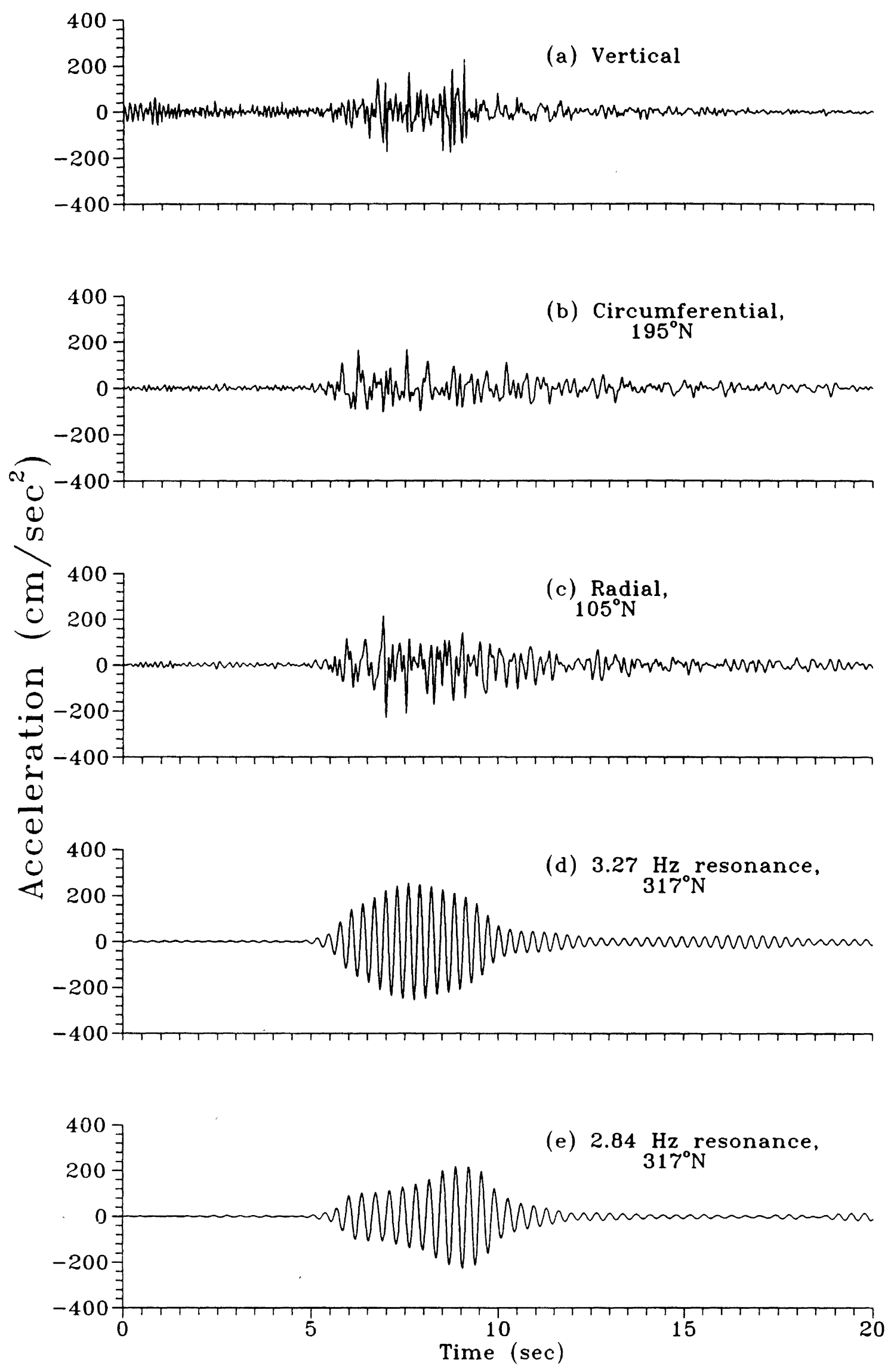

Figure 2 The five components of the Cedar Hills Nursery accelerogram recorded during the 1987 October 1 Whittier Narrows earthquake. 
It is therefore reasonable to conclude that the excitation mechanisms for the responses of Figure 2(d) and (e) are quite different, and could be due to different phases of the earthquake waves because the greatest excitations of the two modes occur at different times.

In physical terms, if the wavefronts match the mode shape for a particular mode, that mode will be strongly excited, and it is probable that different modes will have different wavefronts, and will arrive at different times.

\section{SIGNIFICANCE OF THE RESONANT PARTS}

Whatever the origin of the resonant waveforms shown in Figure 2(d) and (e), their coincidence in direction and similarity in frequency allows them to combine as shown in Figure 3, beating together to result in a large peak acceleration. If these resonances had acted in different directions their resultant would have been less, and if their frequencies had not been so similar there would have been fewer peaks in a beat period, and a smaller chance of individual peaks coinciding to result in a large amplitude at some time. Obviously it is very important to determine why the resonant modes act in the same direction, and to a lesser extent why they have similar frequencies.

\section{POSSIBLE ORIGIN OF THE RESONANCES}

Other accelerograms recorded close to the Cedar Hills Nursery do not show the resonances observed at that site, so the resonances must be site related on a scale of less than a few kilometres. Such resonances are usually attributed to topographic or soil effects, but in this case there is insufficient geotechnical information available to choose between these mechanisms.

The accelerograph site is described both as "in a region of low rolling hills between the alluvial San Fernando Valley and the Santa Monica Mountains. The site is underlain by shallow soil over siltstone. (Soil depth has been estimated to be less than $10 \mathrm{~m}$, but geophysical studies have not yet been performed.)" [2], and "located at the mouth of a canyon on about $10 \mathrm{~m}$ of alluvium" [1]. Thus the observed response could be due to either ridge or basin resonance.
Furthermore, the occurrence of two nearly degenerate modes is consistent with either basin or ridge resonance. However the coincidence in direction of the two resonances, coupled with the lack of vertical resonant motion, is less consistent with basin resonance than with excitation of the first symmetric and antisymmetric transverse modes of a ridge.

This consistency may be demonstrated by considering the simple case of a ridge in the form of a rectangular parallelepiped, $500 \mathrm{~m}$ long, $200 \mathrm{~m}$ wide, and $100 \mathrm{~m}$ high, formed of material having an s-wave velocity of $1000 \mathrm{~m} / \mathrm{s}$ and a p-wave velocity of $1500 \mathrm{~m} / \mathrm{s}$, as shown in Figure 4(a). Resonant frequencies for motions across the length of the ridge are easily determined. The first symmetric shear mode (Figure 4(b), both ends of the ridge moving in phase) has a frequency of $2.5 \mathrm{~Hz}$, the first antisymmetric shear mode (Figure 4(c), the ends of the ridge moving in opposition) has a frequency of 2.7 $\mathrm{Hz}$, and the first cantilever mode (Figure 4(d), both ends of the ridge moving in phase but the ridge bending vertically) has a frequency of $8.8 \mathrm{~Hz}$. Motion observed at one end of the ridge would have many similarities to the Cedar Hills Nursery record, being horizontal, double resonant with a similar separation of frequencies, and with both resonances occurring along the same direction. This idealised ridge is not intended to represent the actual situation at the Cedar Hills Nursery, for which a lower height, lower shear wave velocity and different cross section, may be more appropriate.

In physical terms, an arriving phase which tends to simultaneously push one end of the ridge and pull the other end will excite the higher frequency antisymmetric mode, while an arriving phase which tends to simultaneously push both ends of the ridge will excite the lower frequency symmetric mode (and incidently the cantilever mode). Such different excitations can be caused wave-passage effects. A wave which passes quickly across the ground surface will excite nearby points in phase, but a slower wave will act in opposite senses on points separated by a half wavelength.

The use of the preceding illustration is not meant to imply that such a simple idealised model represents the topology of the Cedar Hills Nursery, but to show that the response of a ridge can in general have features consistent with those seen at the Cedar Hills Nursery.

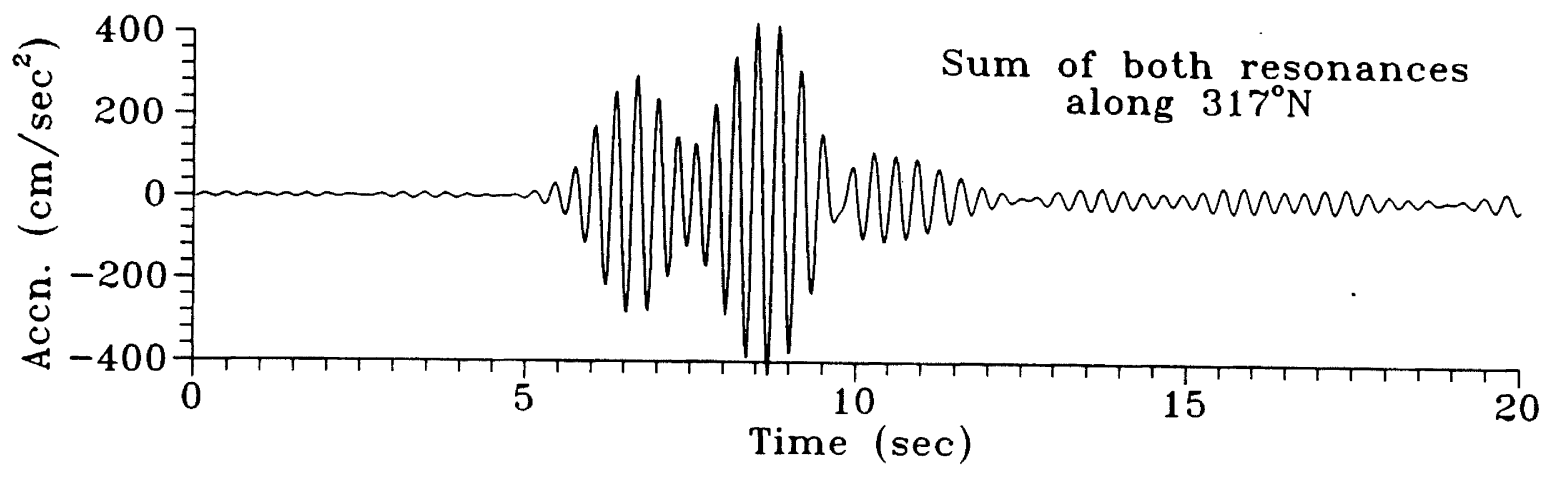

Figure 3 Sum of the $2.84 \mathrm{~Hz}$ resonance and the $3.27 \mathrm{~Hz}$ resonance, both along $317^{\circ}$. 
(d)

(c)

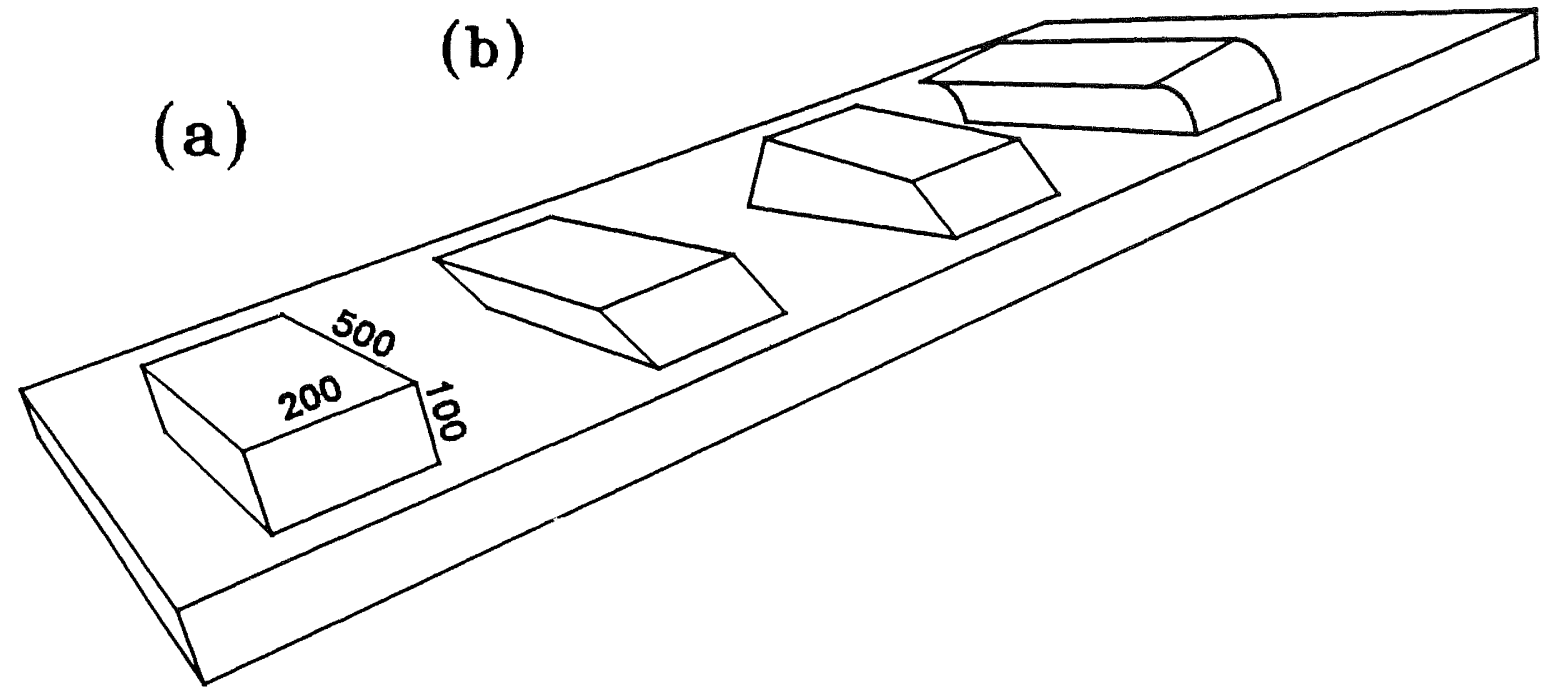

Figure 4 The example ridge. (a) Undeformed ridge with dimensions in metres. (b) Symmetric mode. (c) Antisymmetric mode. (d) Cantilever mode.

\section{NORTHRIDGE EARTHQUAKE}

At the time of writing, the 1994 January 17th Northridge earthquake had just occurred, about $7 \mathrm{~km}$ from the Cedar Hills Nursery site. The accelerogram recorded at the site was only available in analogue form as transmitted by fax machine, and it is not clearly interpretable because of the high frequencies and large accelerations present. Despite these difficulties, resonant behaviour at around $3 \mathrm{~Hz}$ and along a roughly northwest - southeast line is once again seen. This offers further evidence that the resonances are stable and related to local geology. The excitation of these resonances on two occasions by damaging earthquakes, coupled with the lack of excitation during non-damaging earthquakes, suggests that the ability to excite these resonances is somehow related to earthquake magnitude or intensity.

\section{CONCLUSION}

The response of the Cedar Hills Nursery site to the 1897 October 1 Whittier Narrows earthquake can be expressed as the sum of five components. Three of these are conventional vertical, radial and circumferential motions with peak accelerations of a'out $0.2 \mathrm{~g}$, while the other two are resonances along the direction $317^{\circ} \mathrm{N}$, with nearly identical resonant frequencies and with peak accelerations of about $0.3 \mathrm{~g}$. These latter components may be transverse ridge resonances, and are excited in quite different ways.

\section{ACKNOWLEDGEMENT}

The work described in this note was funded solely by the New Zealand Foundation for Science, Research and Technology.

\section{REFERENCES}

1. Campbell, K.W. 1988. The Whittier Narrows, California Earthquake of October 1, 1987 - Preliminary Analysis of Peak Horizontal Acceleration. Earthquake Spectra 4(1):115-137.

2. Shakal, A., Huang, M.J. and Cao, T.Q. 1988. The Whittier Narrows, California Earthquake of October 1, 1987 - CSMIP Strong Motion Data. Earthquake Spectra 4(1):55-100. 